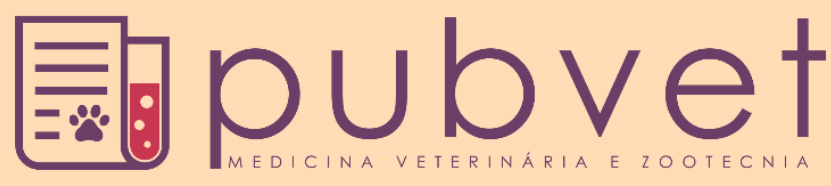

https://doi.org/10.31533/pubvet.v16n01a1015.1-7

\title{
Manejo nutricional em cães diabéticos: revisão
}

\author{
Catia Helena de Almeida Lima Massari ${ }^{1^{*}} \mathbb{C D}^{\bullet}$, Letícia Ribeiro Freires ${ }^{2} \mathbb{D}^{\circ}$, Hugo Leonardo

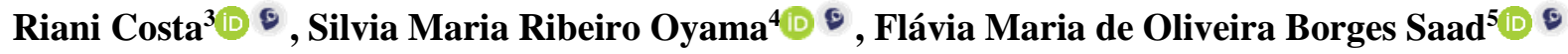 \\ ${ }^{I}$ Professora Colaboradora (PART) do Departamento de Cirurgia, Universidade de São Paulo, São Paulo-SP, Brasil \\ ${ }^{2}$ Graduanda em Medicina Veterinária, Centro Universitário de Paulínia, Paulínia-SP, Brasil \\ ${ }^{3}$ Professor do Curso de Medicina Veterinária, Centro Universitário de Paulínia, Paulínia-SP, Brasil \\ ${ }^{4}$ Pró-Reitora e Coordenadora do Curso de Enfermagem, Centro Universitário Campo Limpo Paulista, Campo Limpo Paulista-SP, Brasil \\ ${ }^{5}$ Professora do Departamento de Zootecnia, Universidade Federal de Lavras, Lavras-MG, Brasil \\ *Autora para correspondência, E-mail: catia.massari@usp.br
}

Resumo. O diabetes mellitus (DM) consiste em um distúrbio metabólico caracterizado por hiperglicemia persistente. Embora a maioria dos cães diabéticos apresentem o diabetes mellitus do tipo 1 , o diabetes mellitus do tipo 2 também pode afligir especialmente aqueles obesos. O presente trabalho tem como objetivo investigar como o manejo nutricional pode auxiliar na terapia do DM2 canino em relação à modulação do processo inflamatório em cães obesos. Realizou-se uma revisão de literatura a partir das seguintes palavras-chave: diabetes mellitus, insulin resistance, hyperglycemia, adipokine, adiponectin, inflammation, obesity, dogs. O presente trabalho conclui que o manejo nutricional é uma ferramenta imprescindível na terapia do DM2 canino, uma vez que se controlando a hiperglicemia, pode-se mitigar as consequências do processo inflamatório desencadeado em pacientes obesos. Portanto, as dietas ricas em proteínas e em fibras são benéficas no aumento da massa magra corporal e na redução da hiperglicemia pós-prandial, gerando bem-estar animal e favorecendo a qualidade de vida em pacientes caninos.

Palavras-chave: Diabetes mellitus, obesidade, nutrição, inflamação, sobrepeso

\section{Nutritional management in diabetic dogs: review}

\begin{abstract}
Diabetes mellitus (DM) is a metabolic disorder characterized by persistent hyperglycemia. Although most diabetic dogs have type 1 diabetes mellitus, type 2 diabetes mellitus can also especially afflict those who are obese. This study aims to investigate how nutritional management can help in the therapy of canine DM2 in relation to the modulation of the inflammatory process in obese dogs. A literature review was carried out using the following keywords: diabetes mellitus, insulin resistance, hyperglycemia, adipokine, adiponectin, inflammation, obesity, dogs. The present study concludes that nutritional management is an essential tool in the therapy of canine DM2 as controlling hyperglycemia can mitigate the consequences of the inflammatory process triggered in obese patients. Therefore, diets rich in protein and fiber are beneficial in increasing lean body mass and reducing postprandial hyperglycemia, generating animal welfare and favoring quality of life in canine patients.
\end{abstract}

Keywords: Diabetes mellitus, obesity, nutrition, inflammation, overweight

\section{Manejo nutricional en perros diabéticos: revisión}

Resumen. La diabetes mellitus (DM) es un trastorno metabólico caracterizado por hiperglucemia persistente. Aunque la mayoría de los perros diabéticos tienen diabetes mellitus tipo 1, la diabetes mellitus tipo 2 también puede afectar especialmente a los obesos. 
Este estudio tiene como objetivo investigar cómo el manejo nutricional puede ayudar en la terapia de la DM2 canina en lo que se refiere a la modulación del proceso inflamatorio en perros obesos. Se realizó una revisión de la literatura utilizando las siguientes palabras clave: diabetes mellitus, resistencia a la insulina, hiperglucemia, adipocina, adiponectina, inflamación, obesidad, perros. El presente estudio concluye que el manejo nutricional es una herramienta fundamental en la terapia de la DM2 canina, ya que el control de la hiperglucemia puede mitigar las consecuencias del proceso inflamatorio desencadenado en pacientes obesos. Por tanto, las dietas ricas en proteínas y fibra son beneficiosas para aumentar la masa corporal magra y reducir la hiperglucemia posprandial, generando bienestar animal y favoreciendo la calidad de vida en los pacientes caninos.

Palabras-clave: Diabetes mellitus, obesidad, nutrición, inflamación, sobrepeso

\section{Introdução}

O diabetes mellitus (DM) consiste em um distúrbio metabólico caracterizado por hiperglicemia persistente, decorrente de deficiência na produção de insulina, na sua ação, ou em ambos os mecanismos. Tal persistência de hiperglicemia está associada a complicações inflamatórias crônicas micro e macrovasculares, aumento de morbidade, redução da qualidade de vida e elevação da taxa de mortalidade ( $\underline{\mathrm{SBD}, 2019}$ ). A classificação do DM baseia-se em sua etiologia (Quadro 1) e, dentre as endocrinopatias, esta é a que mais afeta os pequenos animais (Faria, 2007; Pöppl \& González, 2005).

Quadro 1. Classificação etiológica do diabetes mellitus

\begin{tabular}{|ll|}
\hline \begin{tabular}{ll|} 
Tipo de diabetes \\
mellitus
\end{tabular} & Características \\
\hline $1 *$ & $\begin{array}{l}\text { 1A: deficiência de insulina por destruição autoimune das células } \beta \text { comprovada por exames laboratoriais } \\
\text { 1B: deficiência de insulina de natureza idiopática }\end{array}$ \\
\hline 2 & Perda progressiva de secreção insulínica combinada com resistência à insulina \\
\hline Gestacional & Hiperglicemia de graus variados diagnosticada durante a gestação, na ausência de critérios de DM prévio \\
\hline $\begin{array}{l}\text { Outros tipos de } \\
\text { diabetes mellitus }\end{array}$ & $\begin{array}{l}\text { Neonogênico } \\
\text { Secundário a outras endocrinopatias, doenças do pâncreas exócrino, infecções, síndromes paraneoplásicas } \\
\text { ou administração de medicamentos (hormonais e glicocorticoides) }\end{array}$ \\
\hline $\begin{array}{l}\text { Fonte: Adaptado de American Diabetes Association (ADA, 2019). *O DM tipo 1 subdivide-se em DM tipo 1A e DM tipo 1B, } \\
\text { a depender da presença ou da ausência laboratorial de autoanticorpos circulantes, respectivamente. }\end{array}$
\end{tabular}

A maioria dos cães diabéticos apresenta o diabetes mellitus do tipo 1 (DM1), caracterizado pela destruição das células $\beta$ nas ilhotas pancreáticas, com perda progressiva e, eventualmente, completa da secreção insulínica. O paciente com este tipo de diabetes é conhecido como insulinodependente (Nelson \& Couto, 2015). Embora a fisiopatologia deste distúrbio ainda não esteja totalmente esclarecida, sabese que envolve, além da predisposição genética, fatores ambientais (por exemplo: infecções virais, componentes dietéticos) que desencadeiam uma resposta autoimune no animal.

Já no diabetes mellitus do tipo 2 (DM2), a quantidade de insulina produzida pelo pâncreas pode ser insuficiente e os tecidos corporais são relativamente resistentes a esse hormônio. O paciente com este tipo de diabetes é conhecido como não-insulinodependente e, na maioria das vezes, a resistência insulínica está atrelada à obesidade (Denyer et al., 2021).

Tanto o DM1 como o DM2 são doenças autoimunes, poligênicas, que cursam com quadro de inflamação crônica (com duração de anos), em que inflamação ativa, destruição tecidual e reparação por fibrose ocorrem simultaneamente, além de uma perfusão deficiente que também dificulta a cura de possíveis lesões cutâneas (Ganesh \& Ramkumar, 2020). Os sinais clínicos clássicos de hiperglicemia são poliúria, polidipsia e polifagia (Kumar, 2010).

Sabe-se que a obesidade vem aumentando de forma significativa na população canina nos últimos anos e esse processo é responsável por aumentar as chances do desenvolvimento de distúrbios hormonais e metabólicos, visto que a obesidade tem uma estreita relação com a ativação do processo inflamatório e do estresse oxidativo (André et al., 2017; Blees et al., 2020; Chapman et al., 2019; Penell et al., 2019). Os cães obesos podem desenvolver resistência insulínica e aumento de triglicérides predispondo-os ao 
DM2, e isso se deve ao fato de que as adipocinas, que são proteínas secretadas pelo tecido adiposo, desempenham um importante papel na resposta imunológica e participam da inflamação com suas diversas funções metabólicas e endócrinas (Blees et al., 2020; Verkest, 2014).

Com isso, aumentando a secreção de citocinas pró-inflamatórias, como fator de necrose tumoral alfa (TNF- $\alpha$ ) e interleucinas (IL-6 e IL-1), ocorre ativação da cascata de inflamação, desencadeando um processo inflamatório crônico e resistência tecidual ao hormônio hipoglicemiante. Dessa maneira, a obesidade canina possui associação com diferentes condições de saúde dos cães, como pancreatite, dislipidemia, resistência insulínica e, consequentemente, DM2 (Fragua et al., 2015; Guadarrama-López et al., 2014; Park \& Ahima, 2015).

O presente trabalho tem como objetivo investigar como o manejo nutricional pode auxiliar na terapia do diabetes mellitus do tipo 2 canino em relação à modulação do processo inflamatório em cães obesos.

\section{Desenvolvimento}

Realizou-se uma revisão de literatura por meio da base de dados do Google Acadêmico, buscando artigos científicos a partir das seguintes palavras-chave: diabetes mellitus, insulin resistance, hyperglycemia, adipokine, adiponectin, inflammation, obesity e dogs. Adotou-se como critério de inclusão os manuscritos publicados de 2014 a 2021.

Após análise entre pares, optou-se pela exclusão de artigos duplicados e, finalmente, chegou-se a um resultado de 24 artigos utilizados para redigir o presente trabalho. Assim, os artigos dos seguintes autores foram, finalmente, selecionados como ponto de partida dessa pesquisa: André et al. (2017), Bastien et al. (2015), Bhattacharjee et al. (2016), Blees et al. (2020), Chapman et al. (2019), Clifton et al. (2014), Cortese et al. (2019), Foote et al. (2019), Fragua et al. (2015), Ganesh \& Ramkumar, (2020), Godoy et al. (2018), Lakić et al. (2020), Lee \& Shao (2014), Miller \& Brines (2018), Muñoz-Prieto et al. (2020), Park et al. (2014), Park \& Ahima (2015), Penell et al. (2019), Piantedosi et al. (2016); Sáinz et al. (2015), Singh et al. (2014), Streeter et al. (2015), Verkest (2014), Williams (2017).

Ainda, mais 14 textos científicos entre livros e manuscritos foram integrados por se tratar de referências tradicionais da pesquisa acadêmica em Ciências Veterinárias.

\section{A modulação do processo inflamatório associado ao diabetes mellitus canino e a importância da nutrição clínica}

Diante dessa pesquisa, verificou-se que o excesso de gordura corpórea apresenta íntima relação com a secreção de citocinas inflamatórias e distúrbios de adipocinas, como a leptina e a adiponectina (Lee \& Shao, 2014; Bastien et al., 2015). A extensa adiposidade aumenta de forma significativa a sinalização pró-inflamatória, sendo considerada um fator predisponente ao desenvolvimento da resistência à insulina. Esta condição ocorre quando os tecidos periféricos perdem sua sensibilidade a tal hormônio hipoglicemiante e, assim, os cães diabéticos, por sua vez, acabam aumentando de forma exacerbada a secreção insulínica como um mecanismo compensatório para manter o nível de glicemia próximo ao valor de referência para a espécie (Park \& Ahima, 2015; Fragua et al., 2015; Streeter et al., 2015; Lakić et al., 2020).

Por ser metabolicamente ativo, o tecido adiposo é responsável pela produção de adipocinas como TNF- $\alpha$, IL-6, IL-1, leptina e adiponectina que tem o papel de controlar a homeostase e os processos inflamatórios. O TNF- $\alpha$ é uma citocina que prejudica a sinalização do receptor de insulina, interferindo negativamente na captação de glicose pelas células dos mais diversos tecidos. Logo, a capacidade das células $\beta$ pancreáticas de secretar insulina vai sendo diminuída com o aumento do TNF- $\alpha$, além deste fator também reduzir a atuação de importantes transportadores de glicose como o GLUT-4 (Bajpai \& Tilley, 2018; Fragua et al., 2015; Lakić et al., 2020; Streeter et al., 2015).

A IL- 6 e a IL-1 são citocinas pró-inflamatórias que agem em conjunto para manutenção do processo inflamatório. Elas se apresentam em quantidades elevadas nos pacientes caninos obesos e o aumento dessas citocinas institui inflamação, estresse oxidativo e ainda mais acúmulo de tecido adiposo corpóreo (Guadarrama-López et al., 2014; Fragua et al., 2015; Park \& Ahima, 2015). Já a leptina, é uma adipocina comumente denominada "hormônio da saciedade" e nos cães obesos há o aumento da sua resistência 
tecidual. Dessa forma, seus níveis séricos são aumentados, causando polifagia e, consequentemente, ainda maior ganho de peso, podendo também estar associada à fisiopatologia da pancreatite (Sáinz et al., (2015; Cortese et al., (2019). Em relação à adiponectina, alguns estudos sugerem que ela não tem um papel claro no contexto da obesidade (Streeter et al., (2015), enquanto outros autores apontam que seus níveis são diminuídos em animais com grande quantidade de massa gorda (Park et al., (2014; Park \& Ahima, (2015; Piantedosi et al., (2016; Muñoz-Prieto et al., (20(20). Acredita-se que essa divergência se deve à influência de outros fatores que interferem na mensuração da adiponectina, como idade, diferentes populações caninas, raças, ambientes e distintos métodos analíticos usados nos delineamentos experimentais (Muñoz-Prieto et al., (20(20). Além disso, os níveis de colesterol, triglicérides e frutosamina encontram-se aumentados em animais obesos, enquanto a produção de proteína $\mathrm{C}$ reativa é inibida pela obesidade e pela resistência à insulina em cães (Veiga et al., (2008).

O DM descontrolado pode gerar disfunção nos leucócitos polimorfonucleares, causando defeitos na resposta imunológica e interferindo em processos de quimiotaxia, fagocitose, liberação de leucotrienos, secreção de enzimas lisossomais e morte bacteriana. Com isso, o paciente diabético torna-se susceptível a infecções secundárias causadas por microrganismos que, muitas vezes, não possuiriam um alto grau de patogenicidade num indivíduo hígido. Então, a hiperglicemia persistente faz com que infecções (como as do trato urinário, por exemplo) se tornem mais perigosas, prolongadas e de difícil controle por meio da administração de antibacterianos (Mnif et al., (2013).

Os cães diabéticos sem tratamento adequado podem comumente desenvolver certas complicações a longo prazo devido à hiperglicemia persistente, por isso o DM apresenta um prognóstico reservado. As oftalmopatias estão entre as complicações mais comuns do DM, pois a hiperglicemia permanente pode causar interferências nos vasos sanguíneos da retina (arteríolas pré-capilares e vênulas pós-capilares), que tendem a se romper com facilidade, podendo ocorrer também descolamento de retina e perda de visão, condição esta denominada retinopatia diabética (Miller \& Brines, (2018). A opacificação da lente, popularmente chamada catarata diabética, acaba se desenvolvendo em condições hiperglicêmicas, devido à atração osmótica de água para a lente do bulbo ocular, a qual se torna opaca (Foote et al., (2019; Williams, (2017; Yang et al., 2020).

Conjuntamente, é comum em cães a ocorrência de nefropatia diabética, que leva a um quadro de proteinúria, redução da taxa de filtração glomerular e fibrose renal, determinando a perda da capacidade funcional dos rins (Bhattacharjee et al., 2016). Já o fígado fica comprometido dado que os hepatócitos possuem papel crucial no metabolismo de carboidratos, lipídeos e proteínas, podendo desenvolver complicações clínicas por conta da hiperinsulinemia e da insulinoresistência, causando hipertrigliceridemia (Giestas et al., 2015). Além disso, neuropatia periférica também pode ocorrer em cães com DM, que acabam apresentando distúrbios em movimentos tanto somáticos como autônomos e perda de sensibilidade dolorosa nas extremidades distais dos membros torácicos e pélvicos (Singh et al., 2014).

Levando-se em consideração as consequências metabólicas surgidas em decorrência do processo inflamatório crônico que ocorre em cães diabéticos, uma nutrição adequada associada à prática de atividade física diária é importante para favorecer o controle da obesidade e a conservação do índice de massa muscular corpóreo. Atualmente, indica-se ao cão com DM uma dieta com alta quantidade de fibras e de proteínas (para evitar a sarcopenia diabética), quantidade moderada à baixa de gorduras e quantidade moderada de amido dando preferência sempre aos carboidratos de baixo índice glicêmico (Clifton et al., 2014; Teixeira \& Brunetto, 2017).

Considerando-se as alterações decorrentes do DM em cães, os objetivos da terapia incluem a restauração das concentrações séricas de glicose normais em jejum, a normalização da frutosamina sérica e a reversão ou a atenuação das complicações inflamatórias crônicas. Em pacientes diabéticos, a suplementação de fibras é benéfica ao controle da doença, pois reduz a velocidade de absorção da glicose do intestino e minimiza as flutuações pós-prandiais da glicemia. Isso certamente permite um melhor controle glicêmico e o controle da obesidade canina (Davidson \& Stabenfeldt, 2014).

Também se faz importante o oferecimento de lipídios que possuem um perfil anti-inflamatório, como o ácido eicosapentaenoico (EPA), o ácido docosapentaenoico (DPA) e o ácido docosahexaenoico (DHA) (Godoy et al., (2018). Tais ácidos graxos ômega-3 podem diminuir a ação de mediadores 
inflamatórios e de citocinas como o TNF- $\alpha$ e a IL-6, melhorando sobremaneira a qualidade de vida do paciente diabético (Guadarrama-López et al., 2014; $\underline{\text { Streeter et al., 2015) }}$

\section{Considerações finais}

O manejo nutricional é imprescindível na terapia do DM2 canino, visto que se controlando a hiperglicemia, pode-se mitigar as consequências do processo inflamatório desencadeado em pacientes obesos. Portanto, as dietas ricas em proteínas e em fibras são benéficas no aumento da massa magra corporal e na redução da hiperglicemia pós-prandial, gerando bem-estar animal e favorecendo a qualidade de vida em pacientes caninos. Contudo, sugere-se continuidade e aprofundamento dos estudos sobre o papel das adipocinas em cães com DM2. Uma vez que estas podem atuar como citocinas, sendo proteínas de baixo peso molecular com diversas funções metabólicas e endócrinas, elas participam da inflamação e da resposta do sistema imune, igualmente atuando como sensores do balanço energético.

\section{Referências bibliográficas}

ADA - American Diabetes Association. (2019). Standards of medical care in diabetes. Diabetes Care, 42(Suppl 1), S124-138.

André, A., Leriche, I., Chaix, G., Thorin, C., Burger, M. \& Nguyen, P. (2017). Recovery of insulin sensitivity and optimal body composition after rapid weight loss in obese dogs fed a high-protein medium-carbohydrate diet. Journal of Animal Physiology and Animal Nutrition, 101, 21-30. https://doi.org/10.1111/jpn.12744.

Bajpai, A. \& Tilley, D.G. (2018). The role of leukocytes in diabetic cardiomyopathy. Frontiers in Physiology, 9, 1547. https://doi.org/10.3389/fphys.2018.01547.

Bastien, B.C., Patil, A. \& Satyaraj, E. (2015). The impact of weight loss on circulating cytokines in Beagle dogs. Veterinary Imunology and Immunopathology, 163, 174-182. https://doi.org/10.1016/j.vetimm.2014.12.003.

Bhattacharjee, N., Barma, S., Konwar, N., Dewanjee, S. \& Manna, P. (2016). Mechanistic insight of diabetic nephropathy and its pharmacotherapeutic targets: an update. European Journal of Pharmacology 791, 8-24. https://doi.org/10.1016/j.ejphar.2016.08.022.

Blees, N.R., Wolfswinkel, J., Kooistra, H.S. \& Corbee, R.J. (2020). Influence of macronutrient composition of commercial diets on circulating leptin and adiponectin concentrations in overweight dogs. Journal of Animal Physiology and Animal Nutrition 104, 698-706. https://doi.org/10.1111/jpn.13285.

Chapman, M., Woods, G.R.T., Ladha, C., Westgarth, C. \& German, A.J. (2019). An open-label randomised clinical trial to compare the efficacy of dietary caloric restriction and physical activity for weight loss in overweight pet dogs. The Veterinary Journal 243, 65-73. https://doi.org/10.1016/j.tvj1.2018.11.013.

Clifton, P.M., Condo, D. \& Keogh, J.B. (2014). Long term weight maintenance after advice to consume low carbohydrate, higher protein diets-a systematic review and meta analysis. Nutrition, Metabolism and Cardiovascular Diseases 24, 224-235. https://doi.org/10.1016/j.numecd.2013.11.006.

Cortese, L., Terrazzano, G. \& Pelagalli, A. (2019). Leptin and immunological profile in obesity and its associated diseases in dogs. International Journal of Molecular Sciences 20, 2392. https://doi.org/10.3390/ijms20102392.

Davidson, A.P. \& Stabenfeldt, G.H. (2014). Controle do desenvolvimento gonadal e dos gametas. In: Klein, B.G. (Ed.), Tratado de Fisiologia Veterinária (Cunningham). Elsevier, Rio de Janeiro, Brasil, pp. 408-415.

Denyer, A.L., Partnership, C.D.G., Catchpole, B. \& Davison, L.J. (2021). Genetics of canine diabetes mellitus part 1: Phenotypes of disease. The Veterinary Journal 105611. https://doi.org/10.1016/j.tvj1.2021.105611.

Faria, P.F. (2007). Diabetes mellitus em cães. Acta Veterinária Brasílica 1, 8-22. https://doi.org/10.21708/avb.2007.1.1.258.

Foote, B.C., Michau, T.M., Welihozkiy, A. \& Stine, J.M. (2019). Retrospective analysis of ocular 
neuropathies in diabetic dogs following cataract surgery. Veterinary Ophthalmology 22, 284-293. https://doi.org/10.1111/vop.12589.

Fragua, V., Barroeta, A.C., Manzanilla, E.G., Codony, R. \& Villaverde, C. (2015). Evaluation of the use of esterified fatty acid oils enriched in medium-chain fatty acids in weight loss diets for dogs. Journal of Animal Physiology and Animal Nutrition 99, 48-59. https://doi.org/10.1111/jpn.12312.

Ganesh, G. V, Ramkumar, K.M., (2020). Macrophage mediation in normal and diabetic wound healing responses. Inflammation Research 69, 347-363. https://doi.org/10.1007/s00011-0(20-01328-y.

Giestas, S., Giestas, A. \& Agostinho, C. (2015). Doença Hepática e Diabetes Mellitus - Uma Relação Bi-Direcional. Revista Portuguesa de Diabetes, 10(4), 158-166.

Godoy, M.R.C., McLeod, K.R. \& Harmon, D.L., (2018). Influence of feeding a fish oil-containing diet to mature, overweight dogs: Effects on lipid metabolites, postprandial glycaemia and body weight. Journal of Animal Physiology and Animal Nutrition 102, e155-e165. https://doi.org/10.1111/jpn.12723.

Guadarrama-López, A.L., Valdés-Ramos, R. \& Martínez-Carrillo, B.E. (2014). Type 2 diabetes, PUFAs, and vitamin D: their relation to inflammation. Journal of Immunology Research 2014. https://doi.org/10.1155/(2014/860703.

Kumar, V. (2010). Robbins \& Cotran - Patologia bases patológicas das doenças. Elsevier Brasil.

Lakić, I., Belić, B., Cincović, M., Potkonjak, A., Trailović, D., Kovačević, Z., (2020). Relationship of Circulating Tumor Necrosis Factor Alpha (TNF- $\alpha$ ) and Insulin Secretion and Resistance in Euglycaemic Dogs. Acta Scientiae Veterinariae 48. https://doi.org/10.22456/1679-9216.100400.

Lee, B. \& Shao, J. (2014). Adiponectin and energy homeostasis. Reviews in Endocrine and Metabolic Disorders 15, 149-156. https://doi.org/10.1007/s11154-013-9283-3.

Miller, E.J. \& Brines, C.M. (2018). Canine diabetes mellitus associated ocular disease. Topics in Companion Animal Medicine 33, 29-34. https://doi.org/10.1053/j.tcam.2018.03.001.

Mnif, M.F., Kamoun, M., Kacem, F.H., Bouaziz, Z., Charfi, N., Mnif, F., Naceur, B. Ben, Rekik, N. \& Abid, M. (2013). Complicated urinary tract infections associated with diabetes mellitus: Pathogenesis, diagnosis and management. Indian Journal of Endocrinology and Metabolism 17, 442. https://doi.org/10.4103/2230-8210.111637.

Muñoz-Prieto, A., Cerón, J.J., Martínez-Subiela, S., Mrljak, V. \& Tvarijonaviciute, A. (2020). A Systematic Review and Meta-Analysis of Serum Adiponectin Measurements in the Framework of Dog Obesity. Animals 10, 1-15. https://doi.org/10.3390/ani10091650.

Nelson, R.W. \& Couto, C.G. (2015). Medicina Interna de Pequenos Animais. Elsevier Editora, Amsterdan.

Park, H.-J., Lee, S.-E., Oh, J.-H., Seo, K.-W. \& Song, K.-H. (2014). Leptin, adiponectin and serotonin levels in lean and obese dogs. BMC Veterinary Research 10, 1-8. https://doi.org/10.1186/1746-6148$10-113$.

Park, H.-K. \& Ahima, R.S. (2015). Physiology of leptin: energy homeostasis, neuroendocrine function and metabolism. Metabolism 64, 24-34. https://doi.org/10.1016/j.metabol.2014.08.004.

Penell, J.C., Morgan, D.M., Watson, P., Carmichael, S. \& Adams, V.J. (2019). Body weight at 10 years of age and change in body composition between 8 and 10 years of age were related to survival in a longitudinal study of 39 Labrador retriever dogs. Acta Veterinaria Scandinavica 61, 1-16. https://doi.org/10.1186/s13028-019-0477-x.

Piantedosi, D., Di Loria, A., Guccione, J., De Rosa, A., Fabbri, S., Cortese, L., Carta, S., Ciaramella, P., (2016. Serum biochemistry profile, inflammatory cytokines, adipokines and cardiovascular findings in obese dogs. The Veterinary Journal 216, 72-78. https://doi.org/10.1016/j.tvj1.2016.07.002.

Pöppl, Á.G. \& González, F.H.D. (2005). Aspectos epidemiológicos e clínico-laboratoriais da diabetes mellitus em cães. Acta Scientiae Veterinariae 33, 33-40. https://doi.org/10.22456/1679-9216.14436.

Sáinz, N., Barrenetxe, J., Moreno-Aliaga, M.J. \& Martínez, J.A. (2015). Leptin resistance and dietinduced obesity: central and peripheral actions of leptin. Metabolism 64, 35-46. https://doi.org/10.1016/j.metabol.2014.10.015. 
SBD - Sociedade Brasileira de Diabetes (2019). Diretrizes da Sociedade Brasileira de Diabetes (20192020. Clannad Editora Científica.

Singh, R., Kishore, L. \& Kaur, N. (2014). Diabetic peripheral neuropathy: current perspective and future directions. Pharmacological Research 80, 21-35. https://doi.org/10.1016/j.phrs.2013.12.005.

Streeter, R.M., Struble, A.M., Mann, S., Nydam, D. V, Bauer, J.E., Castelhano, M.G., Todhunter, R.J., Cummings, B.P. \& Wakshlag, J.J. (2015). The associations between serum adiponectin, leptin, Creactive protein, insulin, and serum long-chain omega-3 fatty acids in Labrador Retrievers. Veterinary Medicine: Research and Reports 6, 103. https://doi.org/10.2147/vmrr.s60478.

Teixeira, F.A. \& Brunetto, M.A. (2017). Nutritional factors related to glucose and lipid modulation in diabetic dogs: literature review. Brazilian Journal of Veterinary Research and Animal Science 54, 330-341. https://doi.org/10.11606/issn.1678-4456.bjvras.2017.133289.

Veiga, A.P.M., Price, C.A., Oliveira, S.T., Santos, A.P., Campos, R., Barbosa, P.R. \& Gonzalez, F.H.D. (2008). Association of canine obesity with reduced serum levels of C-reactive protein. Journal of Veterinary Diagnostic Investigation 20, 224-228. https://doi.org/10.1177/104063870802000214.

Verkest, K.R. (2014). Is the metabolic syndrome a useful clinical concept in dogs? A review of the evidence. The Veterinary Journal 199, 24-30. https://doi.org/10.1016/j.tvj1.2013.09.057.

Williams, D.L., (2017). Effect of oral alpha lipoic acid in preventing the genesis of canine diabetic cataract: a preliminary study. Veterinary Sciences 4, 18. https://doi.org/10.3390/vetsci4010018.

Yang, J., Zhao, S. \& Tian, F. (2020). SP1-mediated lncRNA PVT1 modulates the proliferation and apoptosis of lens epithelial cells in diabetic cataract via miR-214-3p/MMP2 axis. Journal of Cellular and Molecular Medicine, 24, 554-561. https://doi.org/10.1016/j.omtn.2020.02.002.

\section{Histórico do artigo:}

Recebido: 15 de agosto de 2021

Aprovado: 16 de setembro de 2021

Disponível on-line: 12 de novembro de 2021
Licenciamento: Este artigo é publicado na modalidade Acesso Aberto sob a licença Creative Commons Atribuição 4.0 (CC-BY 4.0), a qual permite uso irrestrito, distribuição, reprodução em qualquer meio, desde que o autor e a fonte sejam devidamente creditados. 